\title{
The Relationship of Molecular-Genetic Markers of Chemokines with Clinical Manifestations of Chronic Kidney Disease
}

\author{
${ }^{* 1}$ Elena V. Nekipelova, ${ }^{2}$ Olga N. Novakova, ${ }^{3}$ Tatyana I. Yakunchenko, ${ }^{4}$ Mikhail I. Churnosov, \\ ${ }^{5}$ Nina I. Zhernakova, ${ }^{6}$ Vladimir F. Kulikovskiy \\ 1, 2, 3, 4, 5, 6 Belgorod State University, 85 Pobedy Street, Belgorod, 308015, Russia \\ *Email: litovkina@bsu.edu.ru
}

\section{Received: 21st October 2017 Accepted: 16th November 2017, Published: 31st December 2017}

\begin{abstract}
The article presents the results of studying the relationship of polymorphic variants of chemokine genes (1931A/T CCLA, A/G CXCL11 (rs4512021), 403A/G CCL5, C/G CCL2 (rs2857657), -801G/A CXCL12) with the features of clinical manifestations of chronic glomerulonephritis. It was revealed, that the allele-801 A CXCL12 (OR $=1.85, \mathrm{p}=0.04)$ was the marker for the development of hematuric form of chronic glomerulonephritis.
\end{abstract}

Keywords: Chronic Glomerulonephritis, Chemokines, Polymorphism of Genes, Clinical Forms of Glomerulonephritis.

\section{Introduction}

Chronic glomerulonephritis (CGN) is one of the most common chronic kidney diseases, which come to death of patients, due to the development of renal failure (Wada et al., 2008; Litovkina et al., 2014). Despite the relatively low prevalence of CGN in population, the progressive course of disease is the reason why patients with chronic glomerulonephritis are the main contingent of nephrology departments, hemodialysis and kidney transplantation (Nekipelova et al., 2016). The progression of nephritis and the development of renal failure depend on the activity of the process, which is not always clinically evident. Due to this, CGN is often diagnosed for the first time at the terminal stage of chronic kidney disease.

Platelets and neutrophils play a major role among the cellular mediators of immune destruction of the glomerular renal apparatus, including CGN (Woerkom et al., 2000). Their number in leukocyte infiltration depends on the concentration of tumor necrosis factors, interleukins and chemokines, which induce respiratory explosion of neutrophilic granulocytes. It should also be noted, that among the cytokines, chemokines occupy a special place in the development of immune-inflammatory reactions in case of chronic glomerulonephritis. They are produced by immunocompetent cells, infiltrating the glomerular zone and the interstitial space of damaged nephrons (Wada, 2008; Azmandian et al., 2012). According to the literature data, such chemokines as monocyte chemotactic protein-1 (CCL2) and CCL5factor, regulating the activation of normal $\mathrm{T}$-cell expression and secretion, play an important role in the pathogenesis of CGN (Lin et al., 2009).

Therefore, further investigations, aimed at studying the mechanisms of emergence, development and progression of chronic glomerulonephritis, remain an important task for both modern nephrology and medicine in general. The aim of our work is to study the relationship between polymorphic variants of chemokine genes (+1931A/T CCL4, A/G CXCL11 (rs4512021), -403A/G CCL5, C/G CCL2 (rs2857657), -801G/A CXCL12) and the features of clinical manifestations of chronic glomerulonephritis, namely with various clinical forms of the disease.

\section{Materials and Methods}

Two samples were formed for the research. The control group included 462 people, the group of patients with chronic glomerulonephritis consisted of 238 individuals. The samples of patients and control group included Russian residents of the Central Chernozem Region of Russia, who do not have any kinship. Patients were included to the corresponding group after diagnosis of the disease, confirmed by clinical and laboratory-instrumental methods of examination, on the basis of Nephrology Department of the Belgorod Regional Clinical Hospital. Patients with hypertension and diabetes (in past medical history or identified during the examination) were excluded from the group of patients. All patients signed an informed consent for inclusion in the research and the use of obtained data. The control group consisted of individuals without kidney disease and hypertension.

Venous blood, in the volume of 8-9 ml, taken from the ulnar vein of proband, was used as a material for the study. The recovering of genomic DNA from peripheral blood was carried out by a standard phenol-chloroform extraction method (Miller et al., 1988). The analysis of the studied loci was carried out by the method of polymerase chain reaction of DNA synthesis, using oligonucleotide primers and probes.

Statistical processing of data was carried out using the software packages "STATISTICA for Windows 8.0" and "Microsoft Excel 2007". The criterion $\chi^{2}$ was used for the analysis of correspondence between the observed and expected distributions of genotypes, on the basis of Hardy-Weinberg equilibrium. The analysis of association of alleles and genotypes of the studied DNA markers, with various clinical forms of chronic glomerulonephritis, was carried out using conjugation tables $2 \times 2$, with the calculation of the criterion $\chi^{2}$, with the Yates correction for continuity and odds ratio (OR) with $95 \%$ confidence intervals (CI). 


\section{Result and Discussion}

238 patients with chronic glomerulonephritis and 462 persons from the control group were examined. The control group is fully comparable with the sample of CGN patients by age, nationality and place of birth. Genotyping of five polymorphic markers of chemokine genes was carried out: (+1931A/T CCL4, A/G CXCL11 (rs4512021), -403A/G CCL5, C/G CCL2 (rs2857657), -801G/A CXCL12). Inclusion in the analysis of the above genetic polymorphisms was due to the pathogenetic significance of determined chemokines for CGN (Anders et al., 2010).

The investigation of the allele frequencies of studied polymorphic markers of genes revealed, that the empirical distribution of genotypes corresponds to the theoretically expected, at Hardy-Weinberg equilibrium ( $p>0.05$ ), for all studied loci in the group of CGN patients, and in the control group (Table 1).

Table 1.

Summary Information about the Studied Polymorphisms

\begin{tabular}{lll}
\hline Polymorphism & $\begin{array}{l}\text { Studied } \\
\text { groups }\end{array}$ & Minor allele \\
\hline$+1931 \mathrm{~A} /$ T $C C L 4$ & Case & $+1931 \mathrm{TCCL4}$ \\
$+1931 \mathrm{~A} / \mathrm{T}$ CCL4 & Control & $+1931 \mathrm{TCCL4}$ \\
A/G CXCL11 & Case & GCXCL11 \\
A/G CXCL11 & Control & GCXCL11 \\
-403A/G CCL5 & Case & $-403 \mathrm{~A} C$ CL5 \\
-403A/G CCL5 & Control & $-403 \mathrm{ACCL5}$ \\
C/G CCL2 & Case & G CCL2 \\
C/G CCL2 & Control & G CCL2 \\
-801G/A CXCL12 & Case & $-801 \mathrm{~A}$ CXCL12 \\
-801G/A CXCL12 & Control & $-801 \mathrm{~A}$ CXCL12 \\
\hline
\end{tabular}

Notes: MAF, minor allele frequency; HWE, HardyWeinberg equilibrium. $\mathrm{P}$ values were calculated using the $\chi^{2}$ test.

At the next stage of our work, the study of the relationship between molecular genetic markers and the clinical features of CGN, characterizing the course of the disease, was carried out. The analysis of clinical forms in patients with CGN shows, that the disease is manifested in a mixed form (23.3\%), nephrotic form occurs in $22.7 \%$ of patients, latent and hypertonic forms of chronic glomerulonephritis are observed in 19\% of patients (each form) and $16 \%$ of individuals have a hematological form of CGN.

The comparative study of genotypes frequencies of the studied polymorphic markers of chemokines showed, that patients with hypertensive form of CGN had higher concentration of the genotype -403 GA CCL5 (49.50\%), in comparison with the control group $(29.65 \%, \mathrm{OR}=2.15,95 \%$ CI $1.06-4.33, \chi 2=$ $4.63, \mathrm{p}=0.03)$. At the same time, the highest frequency of genotype GG CCL2 (rs 2857657) was revealed in patients with nephrotic CGN (9.53\%),as in comparison with the population control $(2.22 \%$, $\mathrm{OR}=4.42,95 \%$ CI $1.11-16.36, \chi^{2}=4.61, \mathrm{p}=0.03$ ), as compared to the patients with other clinical forms of CGN (0-5.67\%). However, it should be noted, that these genetic differences of individuals with hypertonic and nephrotic forms of CGN, in case of introduction the Bonferroni correction (it is the correction, minimizing the errors of the first kind, i.e. errors, connected with the obtaining of false positive results) do not reach a statistically significant level $\left(\mathrm{p}_{\text {cor }}=0.09\right)$.

According to the literature sources, it is known, that in the pathogenesis of CGN, a special place belongs to such chemokines as CCL5 and CCL2 (Peterson et al., 2004; Stasikowska et al., 2007). CCL2, activating the tubular cells, causes an increase in the secretion of some pro-inflammatory cytokines, for example interleukin-6. Chemokines are involved in the activation of endothelial cells - they stimulate the synthesis of integrins (ICAM-1) on their surfaces, and provide strong adhesion and transmigration of leukocytes through the endothelium, during the formation of infiltrate (Zarbock et al., 2007). Under the influence of CCL2, there is also the proliferation of smooth muscle cells of the vessels, with the secretion of proinflammatory cytokines by them, promoting the progression of kidney disease, due to vascular damage. HWE CCL5 MAFâk\%)integral modulator of many immunological, allergic and infl $x_{2}^{2}$ matory reactions. It participates in the migration and accumulation of lymphocytes 27 monocytes and eosino. Kilic granulocytes in inflammatory foci. $10 \mathrm{dnd}$ pathologically damaged 43.96 of tissues and organs it is a mediator of angiogendsis (Mehrad et 0.52, 200730.0 This chemokine increases the number of macrophages and T-lymphocytes in damaged kidney structures, 18.5 . secrete a spectrum of pro-inflanimatory cytokines, causing the 1.04 destruction of tubular cells. 05 and surrounding connective tissue eléments, leading to sclerosis of glomeruli and fibrosis of interstitial tissue (Muro et al., 2008). Significant changes in the level of CCL5 in peripheral blood, in case of chronic glomerulonephritis, have been described (Nomura et al., 2007).

The study of the distribution of alleles of polymorphic chemokine loci among patients with different clinical forms of CGN revealed the following features. In patients with a hematuric form of chronic glomerulonephritis, the prevalence of allele -801 A CXCL12 is $27.78 \%$, that is 1.6 times higher than in the control group $(17.14 \%, \mathrm{OR}=1.85$ $95 \%$ CI $\left.1.04-3.30, \chi^{2}=4.44, p=0.04\right)$. Attention is drawn to the fact of significant increase in the concentration of this allele in patients with hematuric form of CGN (27.78\%), compared to patients with other clinical forms of disease (11.90-18.75\%).

It should be noted, that in the available literature sources there is no information about the interrelationships of the locus-801G/A CXCL12 with chronic glomerulonephritis. As a stimulator of B-cell precursors growth, CXCL12causes the increase in the homing of certain types of stem cells to damaged organs (including kidneys), the stimulation of proliferation, increased adhesion and cell mobility in the pathological focus (Karimabad et al., 2015); that 
is important in the development of immuneinflammatory reactions in the glomerular apparatus of kidneys (Graham, 2009).

\section{Conclusion}

Thus, within the framework of the present work, the study of the associations of chemokines polymorphic genetic markers with the features of clinical manifestation of chronic glomerulonephritis was carried out. It was established, that the allele-801 A CXCL12 (OR = 1.85) could be considered as a marker for the development of hematuric form in patients with chronic glomerulonephritis.

\section{References}

[1] Anders, H. J., Sayyed, S.A., Vielhauer, V., 2010.Questions about chemokine and chemokine receptor antagonism in renal inflammation. Nephron. Exp. Nephrol., 114(2): Pp.33-38.

[2] Azmandian, J., Mandegary, A., Saber, A., 2012. Chemokine Receptor 2-V64I and Chemokine Receptor 5-Delta32 Polymorphisms and Clinical Risk Factors of Delayed Graft Function and Acute Rejection in Kidney Transplantation. Iran. J. Kidney Dis., 6(1): Pp. 56-62.

[3] Graham, G. J. D6 and the atypical chemokine receptor family: novel regulators of immune and inflammatory processes. Eur. J. Immunol., 39(2): Pp. 342-351.

[4] Karimabad, M.N., Hassanshahi, G., 2015.Significance of CXCL12 in type 2 diabetes mellitus and its associated complications. Inflammation, 38(2): Pp. 710-7177.

[5] Lin, S.L., Castano, A.P., Nowlin, B.T., Lupher, M.L., Duffield, J.S., 2009. Bone marrow Ly6Chigh monocytes are selectively recruited to injured kidney and differentiate into functionally distinct populations.J. Immunol., 183(10): Pp. 6733-6743.

[6] Litovkina, O.N., Nekipelova, E.V., Sirotina, S.S., Yakunchenko, T.I., Efremova, O.A., Sorokina, I.N., 2014. Polymorphism of Vascular Homeostasis Genes and Progression of Chronic Kidney Disease in Patients with Chronic Glomerulonephritis. Research Journal of Pharmaceutical, Biological and Chemical, 5(5): Pp. 1079-1082.
[7] Mehrad, B., Keane, M.P., Strieter, R. M., 2007. Chemokines as mediators of angiogenesis.Thromb.Haemost., 97(5): Pp. 755-762.

[8] Miller, S.A., Dykes, D.D., Polesky, H.F., 1988. A simple salting out procedure for extracting DNA from human nucleated cells. Nucleic acids research, 16(3): P. 1215.

[9] Muro, M., Marin, L., Torio,A., Pagan, J.A., Alvarez-Lopez, M.R., 2008.CCL5/RANTES chemokine gene promoter polymorphisms are not associated with atopic and nonatopic asthma in a Spanish population. Int. J. Immunogenet., 35(1): Pp. 19-23.

[10] Nekipelova, E.V., Novakova, O.N., Yakunchenko, T.I., Krikun, E.N., Zhernakova, N.I., Efremova, O.A., 2016. Clinical and Genetic Research of Chronic Glomerulonephritis. Research Journal of Pharmaceutical, Biological and Chemical Sciences, 7(6): Pp. 322-3227.

[11] Nomura, S., Ishii, K., Kamitsuji, Y., Uoshima, N., Ishikawa, E., Kitayama, H., Hayashi, K., 2007. Elevation of activated platelet-dependent chemokines in patients with anti-CD20 monoclonal antibody (rituximab)-treated non-Hodgkin's lymphoma.Clin. Appl. Thromb. Hemost., 13(2): Pp. 206-212.

[12] Peterson, K.E., Errett, J.S., Wei, T., 2004.MCP1 and CCR2 Contribute to Non-LymphocyteMediated Brain Disease Induced by Fr98 Polytropic Retrovirus Infection in Mice: Role for Astrocytes in Retroviral Neuropathogenesis. J. Virol., 78(12): Pp. 6449-6458.

[13] Stasikowska, O., Wagrowska-Danilewicz, M., 2007.Chemokines and chemokine receptors in glomerulonephritis and renal allograft rejection. Med. Sci. Monit. 13(2): Pp. 31-36.

[14] Wada, T., Matsushima, K., Kaneko, S., 2008. The role of chemokines in glomerulonephritis. Front. Biosci., 13: Pp. 3966-3974.

[15] Woerkom, J. M., Toorn, D.W., 2000. A domestic fight or something else? Nephrol. Dial. Transplant., 15(8): Pp. 1253-1254.

[16] Zarbock, A., Schmolke, M., Bockhorn, S. G., Scharte, M., Buschmann, K., Ley, K., Singbartl, K., 2007. The Duffy antigen receptor for chemokines in acute renal failure: a facilitator of renal chemokine presentation. Crit. Care Med., 35(9): Pp. 2156-2163. 